\title{
GSK-3 $\beta$ mediates cardiac senescence through inhibition of ULK1 directed autophagy
}

\author{
Inna Rabinovich-Nikitin', Lorrie A. Kirshenbaum ${ }^{1,2}$ \\ 'Department of Physiology and Pathophysiology, The Institute of Cardiovascular Sciences, St. Boniface Hospital Albrechtsen \\ Research Centre, Winnipeg, Manitoba R2H 2A6, Canada. \\ ${ }^{2}$ Department of Pharmacology and Therapeutics Rady College of Medicine, Max Rady Faculty of Health Sciences, University of \\ Manitoba, Winnipeg, Manitoba R2H 2H6, Canada.
}

Correspondence to: Dr. Lorrie A. Kirshenbaum, Department of Physiology and Pathophysiology, The Institute of Cardiovascular Sciences, St. Boniface Hospital Albrechtsen Research Centre Rm. 3016, 351 Taché Avenue, Winnipeg, Manitoba R2H 2A6,

Canada. E-mail: LKirshenbaum@sbrc.ca

How to cite this article: Rabinovich-Nikitin I, Kirshenbaum LA. GSK-3ß mediates cardiac senescence through inhibition of ULK1 directed autophagy. J Cardiovasc Aging 2021;1:14. https://dx.doi.org/10.20517/jca.2021.24

Received: 22 Sep 2021 First decision: 23 Sep2021 Revised: 29 Sep 2021 Accepted: 30 Sep 2021 Published: 9 Oct 2021

Academic Editor: AJ Marian Copy Editor: Xi-Jun Chen Production Editor: Xi-Jun Chen

\begin{abstract}
Cardiac senescence is the progressive decline in cardiac performance resulting from decline in age related structural and metabolic processes. Aging cardiac myocytes exhibit alterations in fatty acid and glucose oxidation metabolism, activation of innate immune signaling, enhanced fibrosis, mitochondrial dysfunction, endoplasmic reticulum stress, DNA damage, senescence-associated secreting phenotype and impaired autophagy. GSK-3ß is an upstream regulator of autophagy through its interaction with ULK1 which regulates the initiation step of autophagy and formation of the autophagosme. Herein, we highlight a novel putative molecular mechanism that functionally links cardiac senescence, hypertrophy and autophagy regulation. Ser9 phosphorylation of GSK-3 $\beta$ is critical for promoting cardiac senescence via reduction in ULK1 phosphorylation at Ser913 and inhibition of autophagy.
\end{abstract}

Keywords: GSK-3ß, senescence, aging, autophagy, ULK1

Cardiac senescence is defined as the progressive decline in cardiac function resulting from deterioration of structural and metabolic processes associated with advanced age. At the cellular level, alterations in fatty acid and glucose oxidation, innate immune signaling, and fibrosis are common underlying features of aging 
and, over time, profoundly influence ventricular remodeling and cardiac performance. In addition, at the molecular level, aged cardiac myocytes exhibit organellar defects that include mitochondrial dysfunction, endoplasmic reticulum stress, DNA damage, and senescence-associated secreting phenotype (SASP) ${ }^{[1]}$.

Recent evidence has shown that autophagy, which is crucial for maintaining cellular and tissue homeostasis by removing damaged proteins and organelles from cells, is functionally impaired with age. Autophagy consists of several sequential steps: initiation, nucleation, elongation, and fusion with lysosomes. The initiation of autophagy includes the formation of the autophagosome, which is regulated by Unc-51 like autophagy activating kinase 1 (ULK1). ULK1 is a serine/threonine kinase that phosphorylates Beclin-1, subsequently leading to the phosphorylation and activation of the Vps34 kinase complex, which gives rise to the autophagosome. Autophagy-related genes allow the autophagosomes to mature and subsequently fuse with the lysosome-associated soluble $\mathrm{N}$-ethylmaleimide-sensitive factor attachment protein receptor (SNARE) to form the autophago-lysosome. Hence, the inhibition or activation of ULK1 can profoundly influence autophagy and the clearance of cellular debris - identifying ULK1 as a critical regulator of the autophagic process. While positive activation of ULK1 is regulated by AMP-activated protein kinase, it is negatively regulated by the mechanistic target of rapamycin (mTOR). mTOR complex 1 (mTORC1), which is comprised of mTOR and regulatory-associated protein of mTOR (Raptor), phosphorylate and inhibit ULK1 kinase activity, thereby inhibiting autophagy ${ }^{[2]}$. Therefore, the decline in cardiac autophagy with aging, results in the accumulation of misfolded proteins and dysfunctional organelles such as mitochondria which impair cardiac function. Consequently, studies have shown that while inhibition of autophagy exacerbates pathologies associated with cardiac senescence, activation of autophagy can restore cardiac function and ameliorate aging-related cardiac dysfunction ${ }^{[3]}$.

Glycogen synthase kinase- $3 \beta$ (GSK-3 $\beta$ ) is a serine/threonine kinase that has a role in regulating autophagy in cardiomyocytes. GSK-3 $\beta$ is active under basal conditions and is inhibited during stress conditions. Inhibition of GSK-3 $\beta$ is achieved through phosphorylation in Ser9. One of the principal kinases that phosphorylate GSK-3 $\beta$ at Ser9 is Akt/PI3K, which is phosphorylated and activated by mTORC2 $2^{[4]}$. Previously, GSK-3 $\beta$ was reported as an upstream regulator of autophagy in adult hippocampal neural stem cells through its interaction with ULK1 via phosphorylation of Ser405 and Ser415 within the GABARAPinteracting region ${ }^{[5]}$. In the heart, GSK-3 $\beta$ was shown to be activated during ischemia but inhibited during reperfusion, suggesting that adaptive activation of GSK-3 $\beta$ during ischemia stimulates autophagy, whereas inhibition of GSK-3 $\beta$ inactivates autophagy during reperfusion. Inhibiting GSK-3 $\beta$ expression during both ischemia and reperfusion was detrimental to cardiac function ${ }^{[4]}$.

Notably, GSK-3 $\beta$ also plays an essential role in senescence. Phosphorylation of GSK-3 $\beta$ and its subsequent inactivation was reported in aged hepatocytes ${ }^{[6]}$. Notably, the GSK-3 $\beta$ promoter was suppressed by C/EBP $\beta$ -

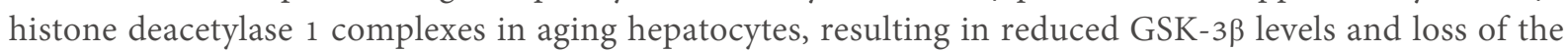
liver's regenerative capacity ${ }^{[7]}$. In the context of the heart, phosphorylation levels of both Akt and GSK-3 $\beta$ were increased during aging, together with the aging-associated increase in mitochondrial permeability pore opening $(\mathrm{mPTP})^{[8]}$. During cardiac hypertrophy, another sine qua non of cardiac senescence, elevated levels of GSK-3 $\beta$ were shown to protect against hypertrophic cardiac myocyte growth. This negative effect of GSK-3 $\beta$ on cardiac hypertrophy may be attributed to transcriptional inhibition of proteins such as $\beta$ catenin, nuclear factor of activated T cells, GATA Binding Protein 4 (GATA4), and eukaryotic initiation factor $2 \mathrm{Be}$. This observation is supported by the fact that overexpression of wild-type GSK-3 $\beta$ or knock-in mutations of Ser9 GSK-3 $\beta$ (GSK-3 $\beta^{\mathrm{S} 9 \mathrm{~A}}$ ) protected the heart from cardiac hypertrophy. Similarly, inhibition of GSK-3 $\beta$ with lithium stimulated the hypertrophic response. Further evidence to support a role for GSK-3 $\beta$ in hypertrophic cardiac growth is derived from studies that showed increased Ser 9 phosphorylation 
of GSK-3 $\beta$ in response to growth agonists, such as endothelin-1, or $\alpha$-or $\beta$-adrenoceptor ligands. These studies revealed a $40 \%-60 \%$ reduction in GSK-3 $\beta$ activity and subsequent increased hypertrophic response in response to growth agonists ${ }^{[9]}$. Together, these studies substantiate that GSK-3 $\beta$ plays an important role in regulating autophagy, cardiac aging, and hypertrophy. However, the molecular mechanism that functionally links these critical processes remains unclear.

A new study by Chen et al ${ }^{[10]}$ in this issue of The Journal of Cardiovascular Aging provides molecular insight into the underlying mechanisms that functionally link cardiac aging, hypertrophy, and autophagy regulation. Through various elegantly designed studies, the authors show that Serg phosphorylation of GSK-3 $\beta$ promotes cardiac senescence via the reduction in ULK1 phosphorylation at Ser913 and inhibition of autophagy.

To better understand the underlying mechanisms of GSK-3 $\beta$ mediated cardiac senescence, Chen et al. ${ }^{[10]}$ first assessed the levels of total and Ser9 phosphorylated GSK-3 $\beta$ in mouse hearts at different ages. Notably, the levels of Ser9 phosphorylated GSK-3 $\beta$ were increased in the hearts of 24 months old mice, compared to the hearts of mice at 6 months of age. This finding was further supported by the observed increased levels of GSK-3 $\beta$ substrates, such as $\beta$-catenin and myeloid cell leukemia 1 in the 24 months old mice, which are known to be increased when GSK-3 $\beta$ is inactivated ${ }^{[11]}$.

Next, the authors examined how the increased phosphorylation of GSK-3 $\beta$ at Ser9 affects cardiac function and survival rates of wild-type (WT) mice and mice in which a mutant form of GSK-3 $\beta$ defective for serine 9 phosphorylation (GSK-3 ${ }^{S 9 A}$ ) was knocked-in $(\beta \mathrm{KI})$ in germ-line. As expected, $\beta \mathrm{KI}$ mice showed higher survival rates than WT mice and coincided with improved cardiac function in the $\beta \mathrm{KI}$ mice. Further, while both systolic and diastolic dysfunction were developed in 24 months old WT mice, the $\beta$ KI mice were found to be protected from age-related cardiac dysfunction, suggesting that increased Ser9 phosphorylated GSK-3 $\beta$ plays a pivotal role in controlling systolic and diastolic cardiac function during aging. In addition, other hallmarks associated with cardiac senescence, including cardiac hypertrophy, fibrosis, apoptosis, and SASP, were significantly increased in WT mice and abrogated in the $\beta$ KI mice. These results highlighted a critical role for Ser9 phosphorylated GSK-3 $\beta$ in promoting senescence in the heart.

Previous studies have shown that autophagy is dramatically decreased with aging, contributing to agerelated pathologies in the heart ${ }^{[1]}$. The findings by Chen et al. ${ }^{[10]}$ support this notion by demonstrating reduced LC3II levels and increased p62 levels in hearts of 24 months old WT mice indicating autophagy impairment. In contrast, $\beta$ KI mice showed higher levels of LC3II and lower levels of p62, suggesting that autophagy was active. Interestingly, overexpression of WT GSK-3 $\beta$ increased LC3II and reduced p62 and stimulated expression of GFP-LC3 puncta, indicating that GSK-3 $\beta$ can activate autophagy in cardiac myocytes.

While studying the molecular mechanism by which GSK-3ß controls autophagy, Chen et al. ${ }^{[10]}$ identified that ULK1 has a consensus amino acid sequence for GSK-3 $\beta$-mediated phosphorylation. To validate that ULK1 was a putative phosphorylation target for GSK-3 $\beta$, the authors performed a kinase assay using recombinant GSK-3 $\beta$ and a kinase-inactive mutant of ULK1 (ULK1K46R). The findings of this assay showed that GSK-3 $\beta$ could directly phosphorylate ULK1. The phosphorylation site in mice was also verified by mass spectrometry, which identified Ser913 as the key serine on ULK1 targeted by GSK-3 $\beta$. Recognizing this specific phosphorylation site led the authors to generate ULK1S913A knock-in mouse. Interestingly, the ULK1S913A mice showed higher levels of p62 and reduced levels of LC3-II, compared to WT mice, demonstrating that phosphorylation of ULK1 at Ser913 plays a pivotal role in regulating GSK-3 $\beta$-induced 


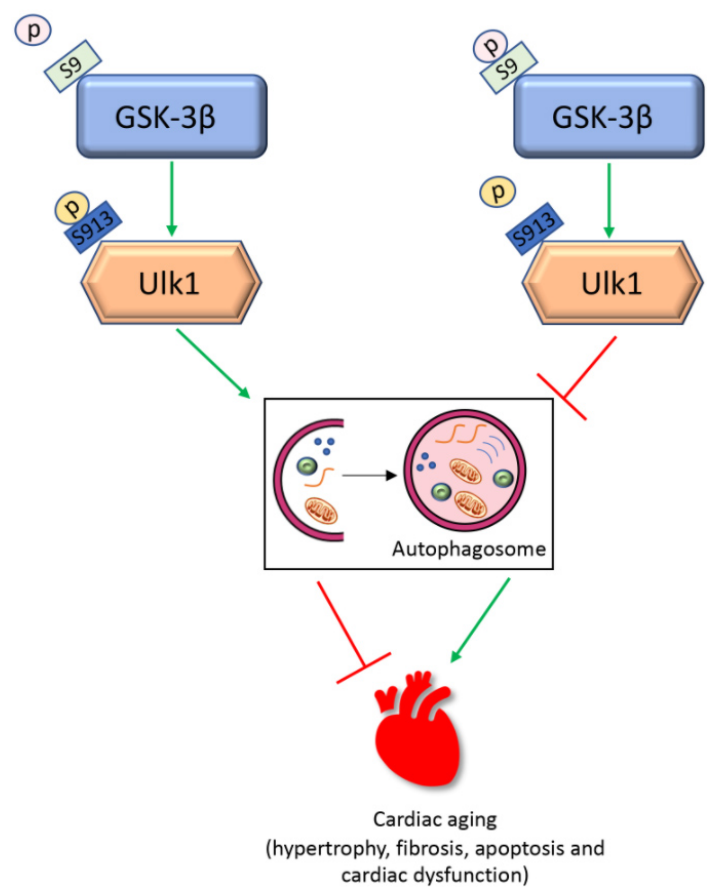

Figure 1. A scheme representing GSK-3 $\beta$ mediated cardiac senescence through inhibition of ULK-1 directed autophagy. Under basal

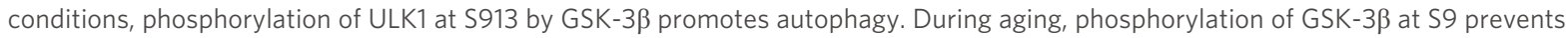
activation of ULK1, leading to inhibition of autophagy and stimulation of cardiac aging processes, including hypertrophy, fibrosis, apoptosis, and cardiac dysfunction.

activation of autophagy in the heart.

While this study provides compelling new evidence that ULK1 phosphorylation at Ser913 by GSK-3 $\beta$ is an essential step for initiation of autophagy during cardiac aging, it also leaves a few open questions that need to be further considered. One question that arises from the study is what happens to the upstream regulators of GSK-3 $\beta$, such as Akt/PI3K and mTORC1/2, during aging? Are these pathways influenced in $\beta K I$ mice through a feed-forward pathway? Furthermore, since $\beta$ KI mutation was germ-line and the GSK$3 \beta^{\text {S9A }}$ mutation is expressed globally and not restricted only to the heart, it may be interesting to investigate the impact of constitutively active GSK-3 $\beta$ in other organs and how the systemic effects of GSK-3 $\beta^{\text {S9A }}$ expressed in other organ systems influence autophagy regulation in the heart. In this regard, since GSK-3 $\beta$ is important in the regulation of glycogen metabolism, cell signaling, and cellular transport, it would be important to determine how the constitutive activation of GSK-3 $\beta$ affects other organs in younger mice and whether other tissues such as skeletal muscle or brain are also resistant to aging through increased autophagy. Likewise, it would be intriguing to explore whether the mechanisms of ULK1 phosphorylation at Ser913 by GSK-3 $\beta$ are similar in other organs or whether the effect of ULK1 is cardiac restricted during aging. It would also be interesting to examine whether other stress conditions which affect GSK-3 $\beta$ activation, such as nutrient stress, and influence autophagy universally use this signaling pathway to activate autophagy or whether this GSK3 $\beta$-ULK1 pathway is limited to aging.

Another crucial question that should be considered arises from a recent study that demonstrated that ULK1 regulates the initiation step of autophagy and the final step of fusion with lysosome and syntaxin $17^{[12]}$. Since Chen et al. ${ }^{[10]}$ assessed autophagy activation and autophagy flux only, it remains unclear whether ULK1 phosphorylation at Ser913 by GSK-3 $\beta$ affects the last step of fusion with lysosome as well. Furthermore, the 
same study showed that phosphorylation of ULK1 enhanced its interaction with heat shock cognate $70 \mathrm{kDa}$ protein (HSC70), therefore increasing its degradation through chaperone-mediated autophagy (CMA) ${ }^{[12]}$. It is intriguing to test whether the mechanism by which GSK-3 $\beta$ regulates autophagy through ULK1 phosphorylation can stimulate CMA also or mainly macroautophagy.

Nevertheless, the study by Chen et al. ${ }^{[10]}$ has revealed a novel and important signaling pathway for autophagy activation during cardiac senescence through ULK1 phosphorylation at Ser913 by GSK-3ß, Figure 1. Addressing the critical signaling pathways and molecular effectors that link autophagy activation by ULK1 may prove beneficial in establishing how modulating autophagy regulation can be applied clinically to curtail cardiac dysfunction and hypertrophy during the inevitable evolution of aging and senescence.

\section{DECLARATIONS}

\section{Authors' contributions}

Collected the data, wrote the paper and revised it: Rabinovich-Nikitin I

Wrote the paper and revised it: Kirshenbaum LA

\section{Availability of data and materials}

Not applicable.

\section{Financial support and sponsorship}

This work was supported by a Foundation grant to Kirshenbaum LA from the Canadian Institute for Health Research (CIHR) and St. Boniface Hospital Foundation, Kirshenbaum LA holds a Canada Research Chair in Molecular Cardiology; Rabinovich-Nikitin I holds a Post-doctoral Fellowship from the CIHR.

\section{Conflicts of interest}

Both authors declared that there are no conflicts of interest.

\section{Ethical approval and consent to participate}

Not applicable.

\section{Consent for publication}

Not applicable.

\section{Copyright}

(c) The Author(s) 2021.

\section{REFERENCES}

1. Tang X, Li PH, Chen HZ. Cardiomyocyte senescence and cellular communications within myocardial microenvironments. Front Endocrinol (Lausanne) 2020;11:280. DOI PubMed PMC

2. Wong PM, Puente C, Ganley IG, Jiang X. The ULK1 complex: sensing nutrient signals for autophagy activation. Autophagy 2013;9:124-37. DOI PubMed PMC

3. Dutta D, Calvani R, Bernabei R, Leeuwenburgh C, Marzetti E. Contribution of impaired mitochondrial autophagy to cardiac aging: mechanisms and therapeutic opportunities. Circ Res 2012;110:1125-38. DOI PubMed PMC

4. Zhai P, Sadoshima J. Glycogen synthase kinase-3ß controls autophagy during myocardial ischemia and reperfusion. Autophagy 2012;8:138-9. DOI PubMed PMC

5. Ryu HY, Kim LE, Jeong H, et al. GSK3B induces autophagy by phosphorylating ULK1. Exp Mol Med 2021;53:369-83. DOI PubMed PMC

6. Souder DC, Anderson RM. An expanding GSK3 network: implications for aging research. Geroscience 2019;41:369-82. DOI PubMed PMC

7. Jin J, Wang GL, Shi X, Darlington GJ, Timchenko NA. The age-associated decline of glycogen synthase kinase 3beta plays a critical role in the inhibition of liver regeneration. Mol Cell Biol 2009;29:3867-80. DOI PubMed PMC 
8. Zhu J, Rebecchi MJ, Tan M, Glass PS, Brink PR, Liu L. Age-associated differences in activation of Akt/GSK-3beta signaling pathways and inhibition of mitochondrial permeability transition pore opening in the rat heart. J Gerontol A Biol Sci Med Sci 2010;65:611-9. DOI PubMed

9. Sugden PH, Fuller SJ, Weiss SC, Clerk A. Glycogen synthase kinase 3 (GSK3) in the heart: a point of integration in hypertrophic signalling and a therapeutic target? Br J Pharmacol 2008;153 Suppl 1:S137-53. DOI PubMed PMC

10. Chen Y, Maejima Y, Shirakabe A, et al. Ser9 phosphorylation of GSK-3 $\beta$ promotes aging in the heart through suppression of autophagy. J Cardiovasc Aging 2021;1:9. DOI

11. Hirotani S, Zhai P, Tomita H, et al. Inhibition of glycogen synthase kinase 3beta during heart failure is protective. Circ Res 2007;101:1164-74. DOI PubMed

12. Wang C, Wang H, Zhang D, et al. Phosphorylation of ULK1 affects autophagosome fusion and links chaperone-mediated autophagy to macroautophagy. Nat Commun 2018;9:3492. DOI PubMed PMC 